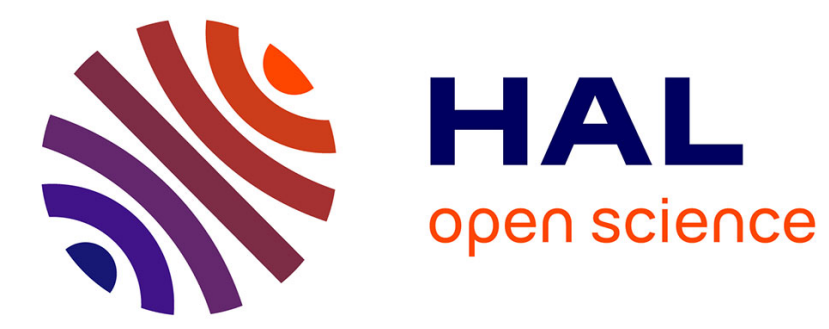

\title{
Cauchy et Bolzano
}

\author{
Hourya Benis Sinaceur
}

\section{To cite this version:}

Hourya Benis Sinaceur. Cauchy et Bolzano. Revue d'Histoire des Sciences, 1973, 26 (2), pp.97-112. 10.3406/rhs.1973.3315 . halshs-01119625

\section{HAL Id: halshs-01119625 https://shs.hal.science/halshs-01119625}

Submitted on 23 Feb 2015

HAL is a multi-disciplinary open access archive for the deposit and dissemination of scientific research documents, whether they are published or not. The documents may come from teaching and research institutions in France or abroad, or from public or private research centers.
L'archive ouverte pluridisciplinaire HAL, est destinée au dépôt et à la diffusion de documents scientifiques de niveau recherche, publiés ou non, émanant des établissements d'enseignement et de recherche français ou étrangers, des laboratoires publics ou privés. 
REV. HIST. SCI.

$\overline{1973-x \times v i / 2}$

\section{Cauchy et Bolzano}

RÉSLMÉ. - On se propose de montrer dans cet article toute la distance qui sépare le style analytique de Bolzano, avec ses tendances logiques profondes qui caractérisent ce qu'on appellera plus tard la rigueur weierstrassienne, et la manière de Cauchy qui reste, malgré des innovations techniques importantes, empêtrée dans le géométrisme traditionnel.

SUMMARY. - The purpose of this article is to show the distance between Bolzano's analytic style with his profond logical tendencies, characteristic of the late called riguor of Weistrass, and the Cauchy's way which remains, despite the important technical innovations, rooted in traditional geometrism.

La mise en rapport de ces deux mathématiciens de la première moitié $\mathrm{du}_{\mathrm{X} \mathrm{x}^{\mathrm{e}}}$ siècle se présente naturellement si l'on se propose de caractériser les normes de rigueur censées avoir apporté, après deux siècles de résultats et de discussions, un "fondement " à l'analyse. Bien que ce "fondement " n'ait pris sa figure définitive, " classique ", qu'avec les travaux de Weierstrass, on en attribue généralement la paternité à A.-L. Cauchy et, en particulier, à son Cours d'analyse de 1821. Or l'histoire révèle que certains des énoncés les plus importants ou les plus typiques de ce Cours, comme la définition de la continuité d'une fonction ou le théorème de convergence connu sous le nom de "critère de Cauchy ", se trouvent déjà dans les écrits de $\mathbf{B}$. Bolzano, plus précisément dans le "Rein Analytischer Beweis..." de 1817 (1). Fait qui pose une double série de questions :

1) Les unes de nature purement historique : priorité d'invention, existence d'un rapport plus ou moins direct entre nos deux auteurs ou leurs œuvres ;

2) Les autres de nature plus générale et de portée épistémologique : une réponse exacte aux questions de la première série

(1) Rein Analytischer Beweis des Lehrsatzes, dass zwischen je zwey Werthen, die ein entgegen gesetzles Resultat gewähren, wenigstens eine reelle Wurzel der Gleichung liegre (Abhandlung. der Kön. Böhm. Gesell. der Wiss., Prąr, 1817 ; traduction française par J. SFвEstik, in lievue d'IIisloire des Sciences, t. XVII, 1964, p. 129-164).

T. XXVI. -1973 
ne contraint-elle pas, par les points qu'elle amène à préciser, à certaine rectification de la conception traditionnellement reçue du développement de l'analyse au xixe siècle?

Tout spécialiste de Bolzano, et plus généralement tout historien des mathématiques au $\mathrm{xIX}^{\mathrm{e}}$ siècle, rencontre nécessairement la première série de questions. Le parallèle entre Cauchy et Bolzano est vieux d'au moins un siècle, puisqu'en $1871 \mathrm{H}$. Hankel, faisant la première mention publique de Bolzano dans l'article "Grenze " du dictionnaire d'Ersch et Gruber (Allgemeine Encyclopädie der Wissenschaften und Künste), écrivait :

"Bolzano a tout pour être placé aussi haut que Cauchy, et il ne lui manque que cet art, si propre au Français, d'apprêter et d'accommoder de la plus agréable des façons ses pensées. Aussi demeura-t-il inconnu et tomba bientôt dans l'oubli ; tandis que Cauchy eut le bonheur d'être considéré comme le réformateur de la science et ses élégants écrits celui d'être rapidement très répandus " (2).

Après lui, O. Stolz, A. Kolman, H. Wussing entre autres ne cessent de rappeler, en même temps que la parenté profonde de Bolzano avec Cauchy, la priorité historique du premier (3). Aujourd'hui voilà de nouveau le parallèle redevenu d'actualité du fait d'un article et d'un livre d'un historien des sciences et de la réponse qu'ils suscitèrent de la part d'un mathématicien (4). Nous aurons quelques remarques à faire en marge de cette polémique; mais réglons d'abord une question évoquée par maint historien de Bolzano : y eut-il un contact personnel antre Bolzano et Ciauchy ?

On sait qu'ils n'entretinrent ni échanges suivis ni correspondance régulière. Bolzano n'est jamais allé à Paris, mais du fond de

(2) Leipzig, 1871, sect. 1, part. 90, p. 185-211.

(3) O. Stouz, B. Bolzano's Bedeutung in der (ieschichte der Infinitesimalrechnung, Mathematische Annalen, XVIII, 1881, p. 255-279; A. Kolmav, Bernard Bolzano, Berlin, Akademie Verlag, 1963 (cet ouvrage rédite en appendice le "Rein Analytischer Beweis ") ; H. Wessisc, B. Bolzano und die (xrundlegung der Infinitesimalrechumg, Zeilschrift für Gieschichle der Naturwissenschaften, Technil und Medizin, I, Heft 3, Berlin, 1964, p. 57-72.

(4) I. Grattan-Ginxiss, Bolzano, Cauchy and the "New Analysis " of the early Nineteenth Century, Archive for History of exact sciences, vol. 6, no 5 , Berlin, 1970, p. 372-400; ibid., The development of the Foundations of malhematical analysis from Euler l" Riemann, Cambridge-Mass., 1970, chap. 3 et 4 ; H. Freudevrinal, Did Cauchy plagiarize Bolzano?, Archive for Hislory of exact Sciences, vol. 7, $\mathrm{n}^{\circ} 5$, nov. 1971, p. $375-392$. 
sa retraite, il tâche de se tenir au courant, est curieux des travaux de Ciauchy dont il lit le: Cours d'analyse, cité explicitement dans son dernier mémoire (1848). Cauchy par contre ne cite jamais Bolzano, semble ignorer ses écrits, mais a séjourné à Prague un certain temps lorsque, ayant pris le chemin de l'exil apres la révolution de Juillet, il accepta le poste de précepteur du duc de Bordeaux, fils aîné de l'ancien roi de France Charles X. On débattit longtemps et contradictoirement la question de savoir si Bolzano put rencontrer Cauchy durant rette période : sa correspondance avec Exner, publiée en 193:) par E. Winter, contient, en effet, des lettres où il exprime à son ami le vœu de rencontrer le mathématicien français. La lettre à Fesl du 18-12-1843 publiée en 1965 dans un nouveau volume de correspondance (5) nous apprend définitivement que ce vou fut exaucé : la rencontre eut lieu vers 18:34-1835, et Bolzano précise même avoir laissé à Cauchy un opuscule, rédigé en français tout exprès à son intention, sur le problème de la rectification des courbes où sont reprises les idées développées dans Die drey Probleme der Rektifikation, Komplanation und Kubierıng (1817). Or, ajoute Bolzano, voilà que Ciauchy publie en 1834, "probablement après avoir lu mon petit essai " un mémoire sur le même sujet; il continue en exprimant sa curiosité à l'égard de ce dernier mais déplore les difficultés qu'il a à se le procurer. De fait il s'agit sans doute du Mémoire sur la rectification des courbes el la quadrature des surfaces courbes imprimé dès 1832, c'est-à-dire bien avant la date de la rencontre! Cette lettre à Fesl n'aurait donc pu justifier le projet de I. Grattan-Guinness de montrer que Ciauchy a plagié Bolzano qu'à la faveur d'une méprise chronologique dont le premier responsable est Bolzano lui-même. Mais que cet indice, le seul explicitement en faveur de la thèse du plagiat, soit en fait négatif, contrarie peu le dessein de Grattan-Guinness; car, de son propre aveu, son argumentation n'est basée sur aucune preuve matériclle mais sur une simple " conjecture historique "; Cauchy connaissait certainement les idées de Bolzano, a su en percevoir la nouveauté et en tirer profit dans ses propres travaux sans éprouver la nécessité de reconnaître publiquement sa dette. Conjecture qui est un pari contre l'évidence, car appuyée non sur des documents mais sur des considérations sociales et psycholo-

(5) E. WINTER, Wissenschaft und Religion im Vormärz. Der Briefwechsel B. Bolzano's mil Michael Joseph Fesl 1822-1848, Berlin, Akademie Verlag, 1965, p. 333. 
giques! L'auteur avoue avoir cherché en vain confirmation dans les archives à notre disposition :

"Il n'y a aucune référence aux cuvres de Bolzano dans les papiers et les lettres de Cauchy; aucune bibliothèque ne garde trace du fait que Cauchy aurait lu ou emprunté une œuvre de Bolzano, et il n'existe, parmi les livres de la bibliothèque personnelle de Cauchy ou du moins ceux qui ont été sauvés de la dispersion et de la perte, ni un exemplaire ni même une copie d'un ouvrage de Bolzano "(6).

Mais Cauchy était un homme bien en place, bien en vue, bien au fait de la production mathématique, habile à exploiter les intuitions et les recherches de mathématiciens socialement moins favorisés ; de même que Fourier aurait inspiré Sur une loi de réciprocité qui existe entre cerlaines fonctions, que les Méthodes de calcul pour les intégrales définies de Legendre seraient à l'origine du Mémoire sur les intégrales définies, de même le "Rein Analytischer Beweis " serait la source du Cours d'analyse, ce que Cauchy pouvait taire d'autant plus facilement que Bolzano, opposant persécuté dans son pays, était réduit à une solitude et à une obscurité bien éloignées des circuits d'échanges du monde scientifique contemporain. Que la Bibliothèque nationale de Paris ait commencé à recevoir les Mémoires de l'Académie de Prague en 1818, précisément à partir du numéro qui contenait le "Rein Analytischer Beweis ", aurait plus servi la perspicacité de Cauchy que la notoriété de Bolzano.

On voit que l'argumentation repose sur des raisons 'xtérieures; quant à l'analyse interne des faits, c'est-à-dire la comparaison précise des énoncés et des ceuvres, elle est sinon absente, du moins très rudimentaire. La mise au point de II. Freudenthal, soutenue par un examen serré des textes, supplée justement ce défaut; aussi les conclusions sont-elles opposées aux idées de I. GrattanGuinness.

Freudenthal dénombre cinq points prétendus communs au "Rein Analytischer Beweis " et au Cours d'analyse :

1) la définition de la continuité d'une fonction;

2) l'énoncé et la démonstration du critère de convergence dit " critère de Cauchy ";

3) l'énoncé et la démonstration du théorème qui constitue le sujet rentral du mémoire de Bolzano;

(6) Archive for History of exacl Sciences, vol. 6, no 5, p. 373. 
4) le théorème de la borne supérieure;

5) la définition des réels.

En ce qui concerne le $5^{\mathrm{e}}$, le $4^{\mathrm{e}}$ et le $2^{\mathrm{e}}$ point nous renvoyons, pour l'essentiel, à l'article de Freudenthal.

Sur la définition des réels, outre le fait que le "Rein Analytischer Beweis " n'y fait aucune allusion, rappelons que le titre sous lequel K. Rychlik a édité en 1962 la portion de la Grössenlehre relative à ce sujet n'est pas de Bolzano lui-même, et qu'après les études de Van Rootselar et de Laugwitz il n'est plus permis d'interpréter univoquement et sans réserve les recherches de Bolzano sur les grandeurs infinies comme une préfiguration de la "théorie des réels " qui a prévalu dans la mathématique cantorienne.

Effet d'une confusion pure et simple entre deux concepts mathématiques bien distincts : celui de borne supérieure d'un ensemble et celui de limite supéricure d'une fonction, le rapprochement établi par Grattan-Guinness au $4^{\mathrm{e}}$ point a encore moins de raison d'être ( 7 ). Cette confusion est sans doute elle-même l'effet d'une deuxième qui consiste à identifier le théorème de la borne supérieure et le théorème dit de Bolzano-Weierstrass et dont l'origine peut se trouver dans une note de la traduction française du "Rein Analytischer Beweis " (op. cit., p. 153, n. 1). Les trois notions ainsi mêlées entretiennent un rapport déterminé dans le seul cas d'un espace métrique $\mathrm{E}$; en effet si $\mathrm{E}$ est métrique, les propriétés suivantes sont alors équivalentes :

I) E est compact ;

II) Toute suite infinie de points de $\mathrm{E}$ a au moins une valeur d'adhérence;

III) Toute partie infinie de $\mathrm{E}$ a au moins un point d'accumulation.

(7) On sait que la borne supérieure d'un ensemble est le plus petit de ses majorants. Le "thérème de la borne supéricure "énonce que tout sous-ensemble de réels, majoré, non vide, possède une borne supérieure. Le concept de limite supérieure d'une fonction est plus complexe en ce sens qu'y intervient déjà un certain nombre de concepts topologiques fondamentaux. Si $f$ est une application d'un ensemble $X$ dans un espace: topologique $Y, \mathscr{B}$ une base de filtre sur $X$, un point $b$ de $Y$ est dil valeur dadherence de $f$ suivant $: B$ si pour tout elément 13 de la base de filtre et pour tout voisinage $V$ de $b, f(B)$ rencontre $V$. La limite supéricure de $f$ suivant $\mathscr{B}$ est la borne supérieure de l'ensemble des valeurs dadhérence de $f$ suivant $\mathscr{B}$ qui coüncide dans ce cas (car l'ensemble des valeurs d'adhérence est un fermé ave le plus grand élément de cet ensemble, appelé l'adhérence de $r$ suivant $\mathscr{B}$.

Enfin, le théoréme de Bolzano-Weierstrass aflirme que toute partie infinie d'un espace compact $E$ a au moins un point d'accumulation dans $E$, ou dans le cas de $\mathbf{R}$ tout sous-ensemble fermé borné posséde au moins un point d'accumulation sur ce fermé borné. 
L'équivalence de I) et de III) constitue le théorème de BolzanoWeierstrass ; II) affirme que l'ensemble des valeurs d'adhérence d'une suite quelconque infinie n'est pas vide; alors si $\mathrm{E} \subseteq \mathbf{R}$ II) implique que l'adhérence de la suite a une borne supéricure et donc que la suite a une limile supérieure.

Cela dit, ni le concept de limite supérieure d'une fonction ou d'une suite ne se trouve dans le "Rein Analytischer Bewcis " ni celui de borne supérieure ne se trouve dans le Cours d'analyse; disjonction si totale qu'elle a suscité plus d'un sarcasme sous la plume de Freudenthal.

Quant au " critère de Cauchy ", l'affaire se résume ainsi : il n'est pas totalement exclu que Cauchy, ayant eu connaissance de l'exposé, confus sur ce point, de son prédécesseur, ait su le tirer au clair et le reformuler de manière plus satisfaisante - re qui ne serait pas un moindre mérite! - mais rien ne le prouve non plus. Freudenthal insiste avec raison sur les différences nombreuses entre la formulation de Bolzano et celle de Cauchy, ce qui exclut de toutes façons l'hypothèse du plagiat grossier. Ajoutons, pour notre part, que d'un côté il s'agit pour Bolzano d'un théorème au sens propre dont il tente de donner une démonstralion mais des applications duquel il ne se préoccupe absolument pas; de l'autre, Cauchy l'énonce non sous forme de théorème, mais simplement et sans démonstration parmi " les considérations générales sur les séries "( $\$ 1$ du chap. VI du Cours) ; par contre suivent de nombreuses applications à la série géométrique, à la série harmonique, à la série $\Sigma \frac{1}{n}$ ! , aux séries alternées, etc. Bon exemple sur lequel se vérifie la différence profonde de style mathématique qui sépare les deux mathématiciens. Bolzano est avant tout préoccupé de rigueur théorique, d'où le souci constant de démonstrations formclles, qui nous semblent aujourd'hui souvent rompliquées, sinon embarrassées et maladroites; d'où encore l'absence d'intérêt pour les résultats et leurs conséquences de quelque nature qu'elles soient. Cauchy, au contraire, donne rarement des démonstrations en forme, recourt souvent à un exemple (cf. la preuve du 3 e théorème du $\$ 3$ du chap. VI) ou à des résultats déjà ronnus par ailleurs. Son exposé a davantage des qualités de synthèse que de rigueur formelle ; jusqu'à un certain point il est même " anti-formaliste " : des résultats, trouvés par des voies différentes ou indépendantes, sont regroupés, reliés entre eux, précisés en ce sens que sont déter- 
minées avec exactitude les conditions dans lesquelles on peut les utiliser sans risque, c'est-à-dire les conditions dans lesquelles leur validité formelle correspond à une situation mathématique concrète, à une réalité analytique, encore bien souvent percue comme une réalité géométrique.

\section{II}

Cela nous conduit à l'essentiel de notre propos qui est de souligner, contre une similitude trop promptement accentuée par nombre d'historiens, la disparité réelle des ouvres de Bolzano et de Cauchy. Non seulement la filiation historique est très improbable mais encore, eût-elle eu le moindre degré de vraisemblance, elle se trouverait annulée par la dissemblance de style de nos deux auteurs. I,étude des $1^{\mathrm{er}}$ et $3^{\mathrm{e}}$ points, parmi les cinq recensés plus haut, étant à cet égard très significative, se placera tout naturellement ici.

Nous avons rappelé, en commençant, que Cauchy est généralement tenu pour le "fondateur " de l'analyse. L'article de Hankel, celui de Stolz, les contributions de Wussing et de Kolman, le livre de (A.-B. Boyer mème (8) tendent, par l'argument de priorité, à faire rejaillir sur Bolzano quelques étincelles de la réputation de Cauchy en leur prêtant une identité de vues (9). La plupart des travaux supposent ainsi l'hypothèse d'un développement monolithique, non différencié, continu, univoque de l'analyse au $\mathrm{xIX}^{\mathrm{e}}$ siècle. Le filon, perdu avec Bolzano, est heureusement retrouvé par Cauchy, qui le redécouvre, l'élargit, l'exploite plus amplement, le transmet à Weierstrass, etc. L'expression "fondement de l'analyse ", à laquelle on substitue souvent après F. Klein celle, encore plus ambiguë d' " arithmétisation de l'analyse ", est investie d'une surdétermination de sens propre à convenir à tous les mathématiciens, de Bolzano à Cauchy, à Riemann, à Weierstrass, à Cantor, etc. C.-B. Boyer écrit, par exemple, "bien que les idées de Bolzano indiquent la direction dans laquelle se réalisa la formulation définitive du calcul... elles n'eurent pas d'influence décisive. Son ouvre passa inaperçue jusqu'à sa redécouverte par H. Hankel, un demi-siècle plus tard. Par bonheur cependant, le mathématicien A. L. Cauchy, poursuivant des idées semblables à peu près au même

(8) The History of the Calculus and its conceplual development, New York, 1949, en particulier le chap. VII.

(9) O. Stolz, cependant, se montre sensible à l'originalité propre de Bolzano. 
moment, réussit à en faire la base du Calcul "(10). Or un examen serré des textes conduit à une rectification locale, relative à Bolzano et Cauchy, qui, si, comme il est probable, elle s'avérait nécessaire ailleurs, modifierait la vision habituelle qu'on a de l'histoire des mathématiques du xix e siècle.

Nous soulignerons tout d'abord que les projels de Bolzano et de Cauchy étaient on ne peut plus distincts. On pourra se reporter, pour s'en convaincre, à la préface du "Rein Analytischer Beweis" et à celle du Cours d'analyse, mais aussi aux "Beyträge zu einer begründeteren Darstellung der Mathematik " (1810) et au "Versuch einer objectiven Begründung der Lehre von den drey Dimensionen des Raumes " (1843). Sur le plan des déclarations de principe seul Bolzano affirme la volonté de fonder les mathématiques (et les titres de ses ouvrages l'attestent suffisamment) c'est-à-dire : de privilégier la démonstration d'un résultat sur le résultat lui-même et sur son exploitation possible, pas seulement pratique d'ailleurs ; le résultat, en tant que tel, ne l'intéresse pas, ce qu'on peut en faire, dans son secteur d'origine ou dans d'autres, n'est pas ce qu'il vise, qu'il puisse avoir des conséquences théoriques ne l'effleure même pas. Ce qui lui importe c'est d'exprimer explicitement et de souligner la nécessité de démontrer les résultats, fussent-ils connus et utilisés depuis longtemps, ce qui l'occupe c'est de tenter pour sa part de faire ces démonstrations.

L'intérêt pour la facture de l'argument qui mène au résultat implique une réflexion sur le concept même de démonstration : le recours à l'exemple, le recours à l'intuition spatiale, le recours aux figures géométriques sont systématiquement rejetés. "Une démonstration géométrique est un cercle vicieux "(11), écrit-il par exemple ; par essence, une démonstration ne peut être qu'analytique.

L'exigence de démontrer rigoureusement se confond donc avec celle de démontrer par des moyens purement analytiques ; et celle-ci n'est qu'une manifestation particulière de la nécessité plus générale de remettre la mathématique sur ses pieds, en procédant toujours du plus formel et du plus abstrait (arithmétique, algèbre, analyse) à ce qui semble à Bolzano, non pas une science indépendante, mais une simple application, une traduction dans l'espace de propriétés

(10) C.-B. Boyer, op. cit., p. 271. C'est nous qui soulignons.

(11) Préface au "Rein Analytischer Beweis ", traduction française, Rev. Hist. Sci., t. XVII, p. 137. 
d'ordre beaucoup plus général : la géométrie. Bolzano a conscience d'une unité profonde de toutes les branches des mathématiques, bâties sur une architecture, une structuration bien déterminées.

Or, pour découvrir cette architecture il faut réfléchir sur le statut des objets mathématiques, sur les concepts, reconnaître ceux qui sont primitifs de ceux qui sont dérivés, de manière à conformer tout exposé à l'ordre logique qui veut qu'on aille du moins au plus dérivé, et s'interroger, enfin, sur la nature ou la définition adéquate des concepts primitifs. Vaste exigence, déjà axiomatique, valable pour toutes les branches des mathématiques puisque la géométrie elle-même, ou plus précisément les Eléments d'Euclide, généralement tenus pour le canon de toute rigueur, ne sont pas exempts de défauts de structure : "Le soubassement de cet édifice par ailleurs somptueux n'est encore ni ferme ni régulier "(12); de même dans les sciences abstraites, encore insuffisamment développées, les concepts de grandeur négative, de grandeur imaginaire, de différentielle, bien que largement employés, ne sont pas encore précisément fixés.

Les exigences de lauchy sont, du moins dans la préface du Cours d'analyse, d'un type opposé ; il ne s'agit ni de fonder, ni de fignoler les démonstrations, ni de refuser l'intuition géométrique, ni essentiellement de définir les concepts premiers de la manière formellement la plus satisfaisante, ni d'envisager une ou plusieurs branches des mathématiques axiomatiquement. Il s'agit tout simplement de faire des mathématiques, c'est-à-dire de résoudre des problèmes et d'améliorer les solutions connues, par restriction, plutôt que par généralisation, en précisant à quelles conditions les formules ne sont pas un symbolisme vide (une série infinie n'est éventuellement l'expression d'une fonction qu'à l'intérieur d'un cercle de convergence dont il faut déterminer le rayon). Nous avons affaire à un type de rigueur essenticllement différent de celui recherché par Bolzano : une rigueur par accumulation et raffinement de savoir, nourrie de tout l'acquis antérieur qu'elle rassemble, enrichit, codifie, clarifie, sans remise en question fondamentale; une rigueur qui est un surplus d'exactitude, fruit d'une systématisation au niveau des résultats, des pratiques et des techniques du calcul, guidée par le souci de mettre en valeur le parti qu'on peut tirer de ces derniers; ainsi le chapitre III du Cours, traitant

(12) Avant-propos des "Beyträge *. 
des "fonctions symétriques et des fonctions alternées " s'intéresse a l' " usage de ces fonctions pour la résolution des équations $\mathrm{du}$ premier degré à un nombre quelconque d'inconnues ", le chapitre VI sur les séries donne des "règles " de convergence, deux termes que nous soulignons (entre autres) pour marquer l'orientation du Cours et la signification de son Introduction, en particulier de la fameuse phrase : "Quant aux méthodes j'ai cherché à lour donner toute la rigueur qu'on exige en géométrie » (13).

A des programmes aussi distincts correspondent naturellement des pratiques bien différentes; ce qui peut se vérifier sur le troisième point supposé commun, dont nous nous étions réservé l'examen. Il faut citer tout l'énoncé du $4^{e}$ théorème du chapitre II pour se rendre compte que Cauchy n'en donne pas de démonstration au sens moderne du terme:

"Si la fonction $f(x)$ est continue par rapport à la variable $x$ entre les limites $x=x_{0}, x=\mathrm{X}$, et que l'on désigne par $b$ une quantité intermédiaire entre $f\left(x_{0}\right)$ et $f(\mathrm{X})$, on pourra toujours satisfaire à l'équation $f(x)=b$ par une ou plusieurs valeurs réelle; de $x$ comprises entre $x_{0}$ et $\mathrm{X}$.

\section{"Démonstration.}

"Pour établir la proposition précédente, il suffit de faire voir que la courbe qui a pour équation $y=f(x)$ rencontrera une ou plusieurs fois la droite qui a pour équation $y=b$ dans l'intervalle compris entre les ordonnées qui correspondent aux abscisses $x_{0}$ et $\mathrm{X}$ : or c'est évidemment ce qui aura lieu (14) dans l'hypothèse admise. En effet, la fonction $f(x)$ étant continue entre les limites $x=x_{0}, x=\mathrm{X}$, la courbe qui a pour équation $y=f(x)$, et qui passe $1^{\circ}$ par le point correspondant aux coordonnées $x_{0}, f\left(x_{0}\right)$, $2^{\circ}$ par le point correspondant aux coordonnées $X, f(X)$, sera continue entre ces leux points : et, comme l'ordonnée constante $b$ de la droite qui a pour équation $y=b$ se trouve comprise entre les ordonnées $f\left(x_{0}\right), f(\mathrm{X})$ des deux points que l'on considère, la droite passera nécessairement entre ces deux points, ce qu'elle ne peut faire sans rencontrer dans l'intervalle la courbe ci-dessus mentionnée " (15).

(13) L'expression "rizueur quion exige en gremetrie ", isolée de son contexte, peut induire en erreur du fait que les mathematiciens soucieux de rigueur, a la fin du xixe siècle, aflirmaient somvent vouloir restaurer la rigueur euclidienne, au sens oì en parlait Bolzano. Nous espérons avoir suffisamment montré que, sous la plume de Cauchy, rigueur géométrique est l'appel non à un formalisme plus strict, mais au contraire au contrôle de sa conformité à une "intuition " des phénomènes tels qu'ils apparaissent dans la pratique familière de l'analyste.

(14) C'est nous qui soulignons.

(15) Cauchy, Cours d'analyse, Paris, 1821 , p. 43-44. 
On le constate, "l'intuition géométrique " est utilisée sans scrupule par le "fondateur de l'analyse" (16), ce qu'en vertu d'une. illusion rétrospective on tend trop souvent à oublier aujourd'hui, mais qu'un historien des mathématiques comme Ph. B. Jourdain remarquait bien en 1905 : "Les conceptions de Cauchy, en analyse ont, sauf sur des points isolés, un sol géométrique " (17). Nous avons déjà noté, par ailleurs, que des vérifications sur exemples se substituent souvent aux démonstrations proprement dites : ainsi pour le théorème sur la convergence d'une série alternée dont le terme général tend vers 0 , où on procède par induction à partir du cas particulier de la série : $1+1 / 2+1 / 3-1 / 4+\ldots$, car " les mêmes raisonnements peuvent évidemment s'appliquer à toutes les séries de ce genre "; ou même qu'aucune tentative de démonstration n'est faite comme dans le cas du "critère de Cauchy " (18). Cauchy, professeur à l'Ecole polytechnique, avait certes plus de raisons de présenter le Cours le plus complet et le plus maniable possible, matrice des nombreux exercices par lesquels se forment les apprentis-mathématiciens, que de s'arrêter à l'imperfection possible. d'une notion isolée. A l'opposé, Bolzano est tellement plus attentif à la signification logique des énoncés qu'à leur fécondité mathématique que la rédaction de ses essais se démarque bien peu de celle des préambules théoriques où se ramasse souvent l'essentiel de son originalité ; l'intérêt se focalise sur des concepts particuliers, détachés de leur ensemble pour être repensés dans un ensemble encore inexistant mais dont apparaissent déjà les impératifs ; c'est consciemment que Bolzano renonce à la publication de " traités complets ", tenant à " faire connaître d'abord... ses concepts, différents des concepts habituels " (19) ; le théorème sur l'annulation d'une fonction continue prenant des valeurs de signes opposés

(16) Lne méthode de démonstration "directe et purement analytique " est rejetéc dans la note II d du Cours. Freudenthal, remarquant qu'elle emploie des instruments tout à fait différents de ceux mis en jeu par Bolzano, conclut que rauchy a emprunte tout au plus à ce dernier l'idee de fournir une demonstration purement analytique.

(17) Ph. B. Jovendx, "The theory of functions with hauchy and rauss ", Biblintheca Malhemalica (3), VI (1905), p. 190-207; citation à la p. 206.

(18) Aussi Freudenthal remarque judicieusement que c'est, dans l'uruvre de cauchy, une découverte prématurée : Cauchy en fait pratiquement usage pour démontrer la convergence de certaines séries, mais l'importance logrique de l'énoncé n'apparaîra que beaucoup plus tard.

(19) Préface du "Rein Analytischer Beweis ", traduction francaise, Rev. Hisl. Sci., t. XVII, p. 141 . 
n'est retenu qu'à cause de l'impact qu'il a au niveau de la conceplualisalion proprement dite.

Nous en arrivons par là à considérer, enfin, la définition de la continuité d'une fonction et l'emploi du concept de limite sur lesquels règne, à notre avis, la plus grande confusion. Faisons brièvement le point :

a) On admet généralement que l'analyse classique repose tout entière sur le concept de limite, qui a supplanté la référence tantôt à l'intuition cinématique ou géométrique d'un point mobile, tantôt à l'idée de quantité infinitésimale, tantôt à l'idée de quantité évanouissante.

b) Mais on dit trop rapidement que c'est Cauchy qui a introduit la "méthode des limites " entendant par là, plus ou moins vaguement, l'emploi systématique de l' "épsilonisation ", c'est-à-dire le recours à une formulation bâtie sur le couple " $\varepsilon-\delta$ ». Par exemple C.-B. Boyer écrit que par opposition aux cuvres de Lacroix, L'Huilier, d'Alembert où il gardait une nature géométrique, le concept de limite " devient dans relle de Cauchy... clairement et définitivement arithmétique »(20), et plus loin :

"Avec Cauchy on peut dire sans hésitation que les concepts fondamentaux du Calcul ont reçu une formulation rigoureuse. C'est pourquoi Cauchy a été communément considéré comme le fondateur du Calcul différentiel exact au sens moderne. C'est sur une définition précise de la notion de limite qu'il construit sa théorie de la continuité et des séries infinies, de la dérivation, de la différentielle el de l'intégrale" (21).

c) Après quoi on identifie sans réserve les contributions de Bolzano et de Cauchy dans ce domaine, disant par exemple que la définition de la continuité par Bolzano, dans le "Rein Analytischer Beweis ", " n'est pas essentiellement différente de celle donnée un peu plus tard par Cauchy..."

Or, 1) l' "épsilonisation " n'est pas l'œuvre de Cauchy, mais celle de Weierstrass ; et 2) la définition de la continuité n'est pas essentiellement identique chez Bolzano et chez Cauchy.

1) Cauchy remet certes en avant l'idée de limite qui devient effectivement la clef de voûte du Cours, mais on ne peut dire qu'il

(20) Op. cil., p. 272.

(21) Ibid., p. 282. 
en donne une définition purement arithmétique ou purement analytique. Sa définition :

"Lorsque les valeurs successivement attribuées à une même variable s'approchent indéfiniment d'une valeur fixe, de manière à en différer aussi peu que l'on voudra, celte dernière est appelée limile de toutes les autres " (22)

n'enveloppe pas moins d'intuition géométrique que celle contenue dans le Traité de Lacroix par exemple (23). Le mérite de Cauchy est d'ordre heuristique et non conceptuel : il n'est pas d'avoir formulé rigoureusement les concepts fondamentaux du calcul intégral et différentiel, mais d'avoir réorganisé autour de cette notion de limite, devenue ainsi centrale bien qu'imparfaitement définie, l'ensemble des résultats et des notions voisines et distinctes à la fois et dont on savait mal déterminer le lien : ainsi $d y / d x$ est définie comme la limile du quotient de deux différences, une série est convergente si la suite de ses sommes partielles a une limite, et surtout l'intégrale définie est conçue comme limite d'une somme de produits (les "sommes de Riemann"). Le mérite de Cauchy est d'avoir su offrir une synthèse féconde, ce que Bolzano, procédant par coups de sonde fragmentés, a précisément manqué.

2) Il est en effet déconcertant de constater combien est rigoureux le concept de limite impliqué dans la définition de la continuité (préface du "Rein Analytischer Beweis "), tandis que reste obscure et compliquée la formulation du critère de convergence ( $\$ \S 6$ et 7 ), où une périphrase peu maniable renvoie au concept demeuré sans nom et sans notation symbolique. Si bien que cette fameuse définition de la continuité apparait comme une intuition heureuse et singulière, absolument rigoureuse au sens où, au symbolisme près, elle est purement analytique et ne recourt pas à la notion " d'accroissement infiniment petit " (24), absolument abstraite dans la mesure où elle est explicitement offerte comme le remède à

(22) Cette définition, l'unique du Cours, se trouve dans les Préliminaires.

(23) Traité du calcul integral el différentiel, $2^{\mathrm{e}}$ éd., t. I, 1810, p. 13. Par exemple, la fonction $\frac{a x}{x+a}$ a la quantité a pour limite quand $x$ augmente indéfiniment si "la différence $a-\frac{a x}{a+x}=\frac{a}{x+a}$ devient d'autant plus petite que $x$ est plus grand et peut être rendue moindre qu'aucune grandeur donnée, quelque pelite que soil cette grandeur; en sorte que la fraction proposée peul approcher de a aussi près que l'on voudra".

(24) r.f. le hésumé des legons sur le calcul infinilésimal, 1823. 
$l$ " indistinction " des " roncepts de plusieurs mathématiciens, même réputés ".

Au contraire, la définition de Cauchy, d'une part, ne fait pas l'économie des infiniment petits qui, définis explicitement dans les Préliminaires (25), en fournissent la base immédiate:

"Parmi les objets qui se rattachent a la considération des infiniment petits, on doit placer les nolions relatives a la continuité ou à la discontinuité des fonctions "(26).

\section{Après de brèves explications suit la définition :}

"... la fonction $f(x)$ sera, entre les deux limiles assignées a la variable $x$, fonction continue de cette variable, si, pour chaque valeur de $x$ intermédiaire entre ces limites, la valeur numérique de la différence

$$
f(x+\alpha)-f(x)
$$

décroît indéfiniment avec celle de $\alpha$. En d'autres termes, la fonction $f(x)$ restera continue par rapport à $x$ entre les limites données, si, entre ces limiles, un accroissement infiniment petit de la variable produit loujours un accroissement infiniment pelit de la fonction elle-même " (27) (souligné par (iauchy) (28).

(25) " liemarquons d'abord qu'il l'exemple considéé plus hautj a pour base un concept incorrect de la continuité. Car dans une définition correcte, on entend par l'expression : une fonction $f(x)$ varie suivanl la loi de continuilé pour loules les valeurs de $x$ situées à l'inlerieur ou à l'extérieur de cerlaines bornes, rien d'autre que ceci : si $x$ est une telle valeur quelconque, la différence $f(x+\omega)-f(x)$ peul être rendue plus petite que loute grandeur donnée, si l'on peul loujours prendre $\omega$ aussi pelit que l'on voudra, c'est-à-dire lorsqu'on a (selon les notations que nous avons introduites dans le $\$ 14$ dı "Théorime du binòme ", etc., Prague, 1816): $f(x+\omega)=f(x)+\Omega$.

lin 1817 , Bolzano cherche charement l'élimination de l'infiniment petit, comme en témoigne son essai : "I es trois problemes de la rectification, de la complanation et de la cubature resolus sans consideration de l'infiniment petit, sans l'hypothèse de l'axiome d'Archinède el sans aucune presupposition qu'on ne puisse rigoureusement démontrer ", leipzig, 1817. Plus tard, a l'époque des "Paradoxes de l'Infini " il aura, concernant ce sujet, une position beaucoup plus équivoque.

(26) A.-L. Cincciry, Cours d'analyse, Paris, 1821 , p. 3.1.

(27) Ibid., p. 34-35.

(28) I,es notions de quantité infinie, quantite infiniment petite, infiniment grande sont loin d'être absentes de l'auvre de Gauchy ; les infiniment petits interviennent aussi bien dans la définition de la continuilé que dans celle de l'intérrale définie ; le lours les défnit non comme des quantités fixes mais comme des quantiles variables tendant vers 0 , les variables elles-memes atant des quantites "devant recevoir successivement plusieurs valeurs différentes les unes des autres $"$. L'idée d'infiniment petit est donc résolue en celle, confuse pour nous aujourd'hui, de quantilé variable. A la fin de sa vie, Bolyano, toujours suncieux de clarte logique des concepts, s'apercevra de cette confusion : "Ce que les mathématiciens nomment quantite variable n'est en vérité pas du tout une quantité, mais le simple concept, la simple représentation d'une grandeur qui comprend non une seule, mais un ensemble infini de quantités toutes distinctes les unes des autres par leur valeur, $i$. e. par leur grandeur ", ecrit-il dans un paragraphe des "Paradoxes do l'Infini ", où il vise directement Cauchy. 
D'autre part cette définition n'est pas établic pour elle-même, c'est-à-dire pour la rigueur formelle, mais comme repère destiné à faciliter la tâche de "reconnaître entre quelles limites une fonction donnée de la variable $x$ est continue par rapport à cette variable "; elle ne fournit pas le concept primitif, indispensable pour une démonstration formelle de la continuité d'une fonction $f$ quelconque, mais doit plutôt alimenter l'intuition acquise par le maniement des fonctions usuelles et aider à déterminer pratiquement leurs intervalles de continuité (29).

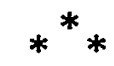

Ces remarques nous conduisent à conclure que les œuvres de Bolzano et de Cauchy représentent deux courants distincts, hétérogènes à l'origine, et qui ne se rencontrent pas avant les années 1870, avec les travaux de Weierstrass, Cantor, etc. Seule l'illusion rétrospective et une connaissance indirecte des textes permettent de les amalgamer dans un même cours ininterrompu supposé traverser tout le $\mathrm{x}_{\mathrm{x}} \mathrm{e}$ siècle mathématique. Bolzano n'explique pas Cauchy, mais il peut certainement être considéré comme un précurseur de Weierstrass, même si c'est, en fait, sous l'impulsion imprimée par Cauchy et à partir de l'héritage qu'il

(29) Remarquons que Cauchy renverse l'ancieme situation en ce sens qu'au lieu d'appréhender l'idée de limite par le biais du "principe de continuité ", il fonde au contraire celui-ci sur celle-là : "M. Poncelet, ecrit-il dans son "Rapport sur le Mémoire relatif aux propriétés projectives ", emploie encorr... ce qu'il appelle le principe de continuité... Ce principe n'est, à proprement parler, qu'une forte induction à l'aide de laquelle on étend des théoremes, établis d'abord à la fiveur de certaines restrictions, aux cas où ces mêmes rostrictions n'existent plus. Néanmoins nous pensons qu'il ne saurait être admis généralement et appliqué indistinctement à toutes sortes de questions en Géométrie, ni même en Analyse... On sait, par exemple, que, dans la détermination des intégrales définies, et par suite, dans l'évaluation des longueurs, des surfaces et des volumes, on rencontre un yrand nombre de formules qui ne sont vraies qu'autant que les valeurs des quantites qu'elles renferment restent comprises entre certaines limites" (in J. V. Po.vcrlet, Trailé des propriéles projectives des figures, Paris, 1822, p. vir-xvi ; passage cité $p . \quad 1 x-x)$. Mais ce texte montre simultanément que l'idée de rigueur poursuivie est bien de même nature que celle dont il est question dans l'Introduction du Cours d'analyse; il s'agit toujours de déterminer l'aire de validité des formules, d'en limiter la portée et d'en restreindre l'application, et non d'exiger, en vertu d'un a priori général, de plier la continuité, voire toute l'Analyse, à des normes purement analytiques. 
a laissé que Weierstrass a développé sa propre manière de faire des mathématiques. La lettre de H. A. Schwarz à G. Cantor du 1 er avril 1870 (30) reconnaît que "la méthode de démonstration (die Schlussweise) de Weierstrass est un développement des principes de Bolzano ", anticipation un demi-siècle oubliée des fondements donnés à l'analyse classique.

$$
\text { C.N.R.S., }
$$

\section{IJourya Sinaceur.}

Paris.

(30) Publié par H. Mrscikowsk, Probleme des Lnendlichen, Werk und Ieben G. Cantors, Braunschweir, 1967. 